\title{
Supporting Information - Hierarchical Markov State Model Building to Describe Molecular Processes
}

David K. Wolfe ${ }^{\dagger}$, Joseph R. Persichetti ${ }^{\dagger \#}$, Ajeet K. Sharma ${ }^{\dagger, \|}$, Phillip S. Hudson ${ }^{\perp}, \nabla, H$. Lee Woodcock $^{\perp}$, Edward P. O’Brien ${ }^{\dagger, \ddagger, \S, *}$

${ }^{\dagger}$ Department of Chemistry, ${ }^{\ddagger}$ Bioinformatics and Genomics Graduate Program, ${ }^{\S}$ Institute for Computational and Data Sciences, Penn State University, University Park, Pennsylvania 16802, United States

"Department of Physics, Indian Institute of Technology, Jammu 181221, India

${ }^{\perp}$ Department of Chemistry, University of South Florida, Tampa, Florida 33620, United States

${ }^{\#}$ Authors contributed equally.

* Corresponding author: epo2@psu.edu

$\nabla$ Present address: Laboratory of Computational Biology, National Heart, Lung, and Blood Institute (NHLBI), National Institutes of Health (NIH), Bethesda, Maryland 20892, United States 


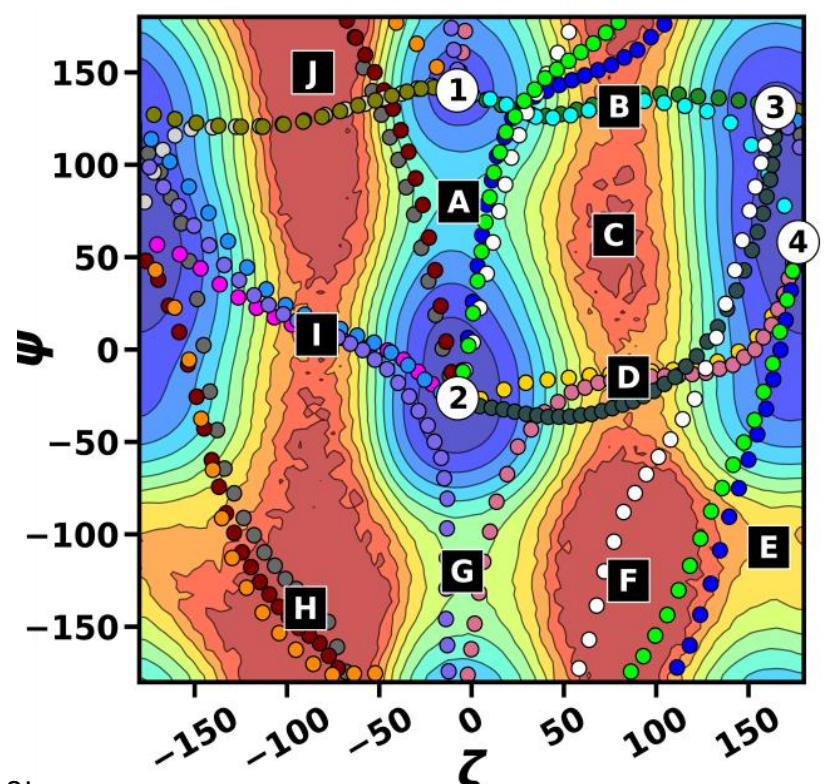

Figure S1. Free-energy sh...un positions of all states are numbered and all transition states considered are lettered. The pathway colors are consistent with the flux legend (Figure S3). 


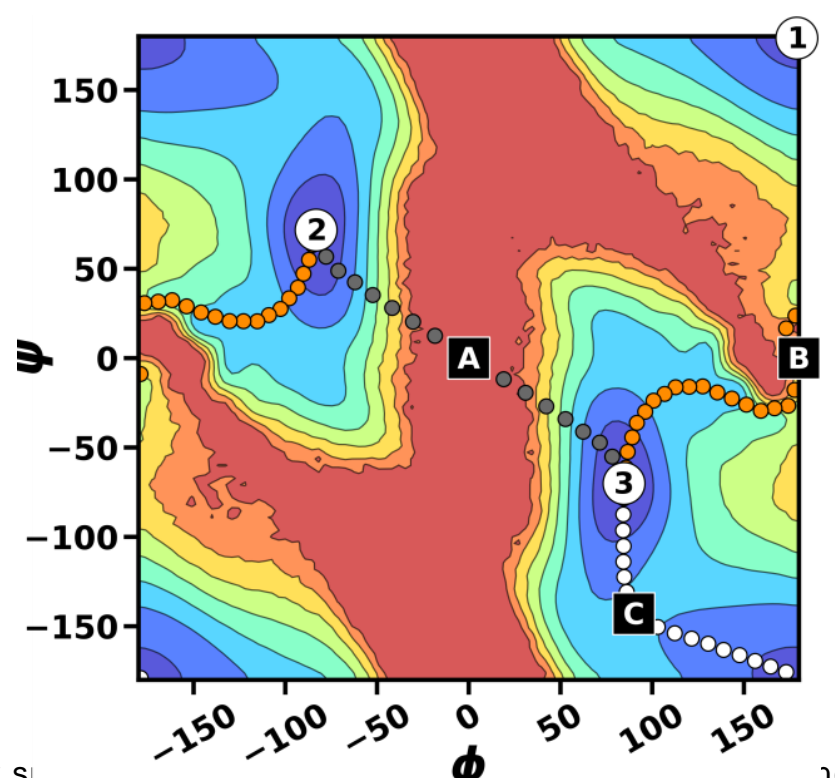

Figure S2. Free-energy $\mathrm{S}$

positions of all states are numbered and all transition states considered are lettered. The following pathway colors apply: 2A3: grey, 1C3: white, and 2B3: orange. 


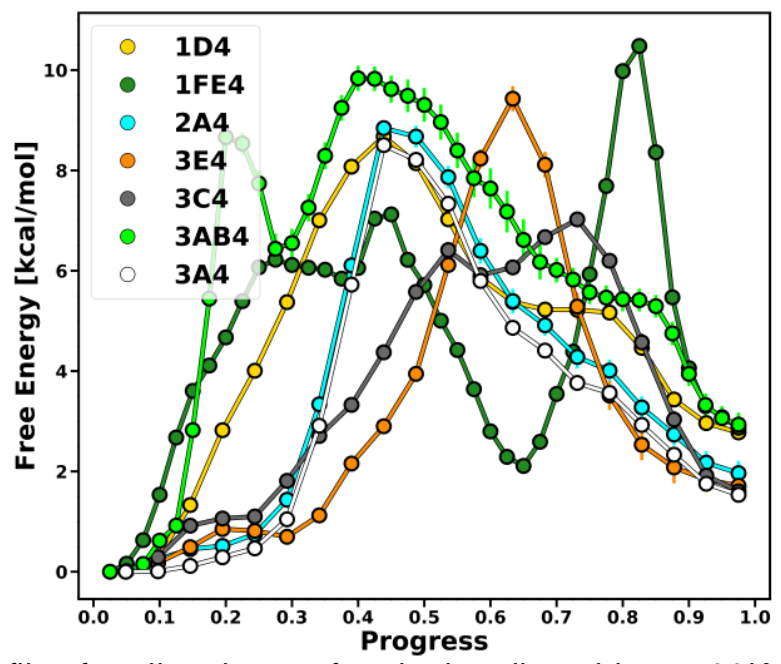

Figure S3. Free-energy profiles for all pathways for alanine dipeptide at $500 \mathrm{~K}$. Error bars represent the $95 \%$ confidence intervals obtained from block averaging the umbrella sampling simulations into 5 evenly sized blocks. 
A

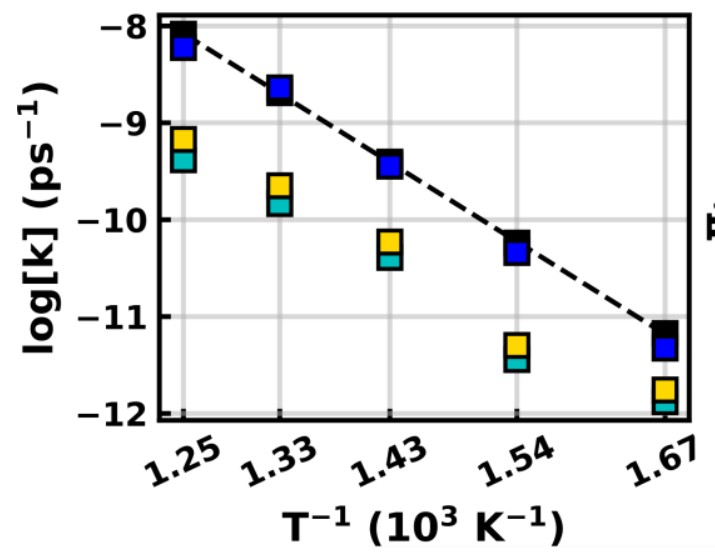

B

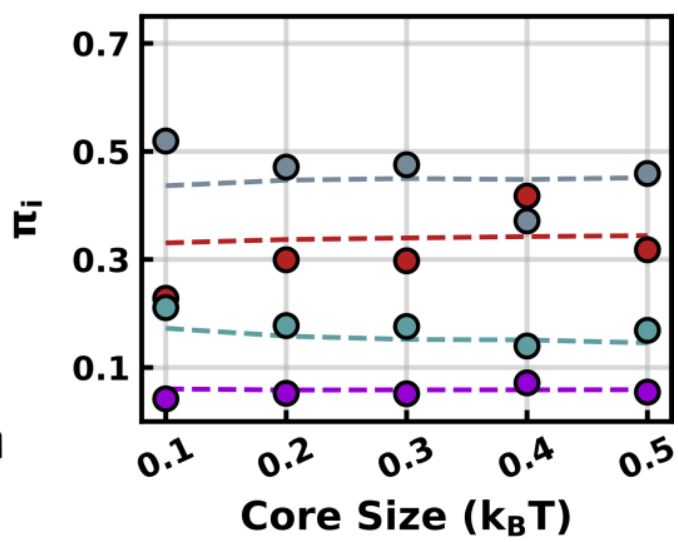

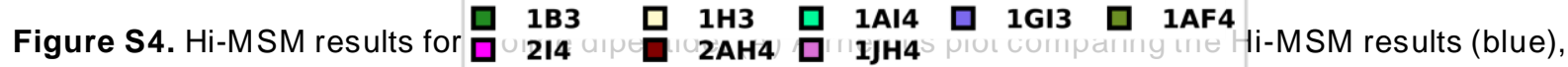
the Hi-MSM with only path 2D4 (cyan), and the singie painway (2D 4 oniy) resulis (gold) to the unbiased simulations (black). The results shown are for the Hi-MSM constructed with a core size of $0.3 k_{B} T$. (B) The probability of occupying states 1-4 at equilibrium calculated with the Hi-MSM (circles) compared to the simulation results (dashed lines added to guide the eye). The color scheme for states 1-4 matches those in Figure 3B. Results shown for the model constructed at 700K. (C) Fraction of the flux from $\Lambda_{1} \rightarrow$ $\Lambda_{2}$ from the Hi-MSM for individual pathways. The results are displayed for the models constructed at a core size of $0.3 k_{B} T$. Due to the pruning procedure for the pathways, not all paths appearing in the legend are present at every temperature. Pathway labels correspond to the Markov and transition state labels shown in Figure S1. The error bars, which are too small to be visible, represent the $95 \%$ confidence intervals obtained from bootstrapping the underlying rate matrices. 


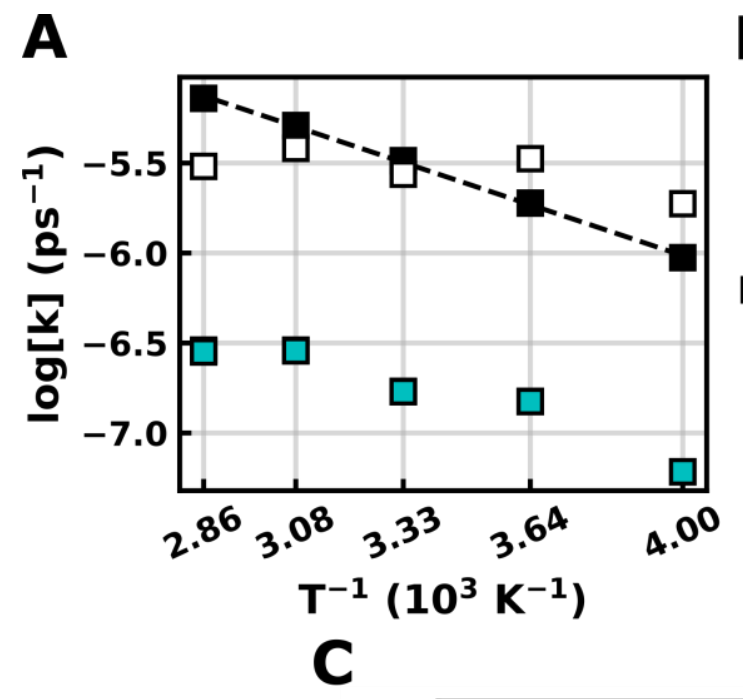

B
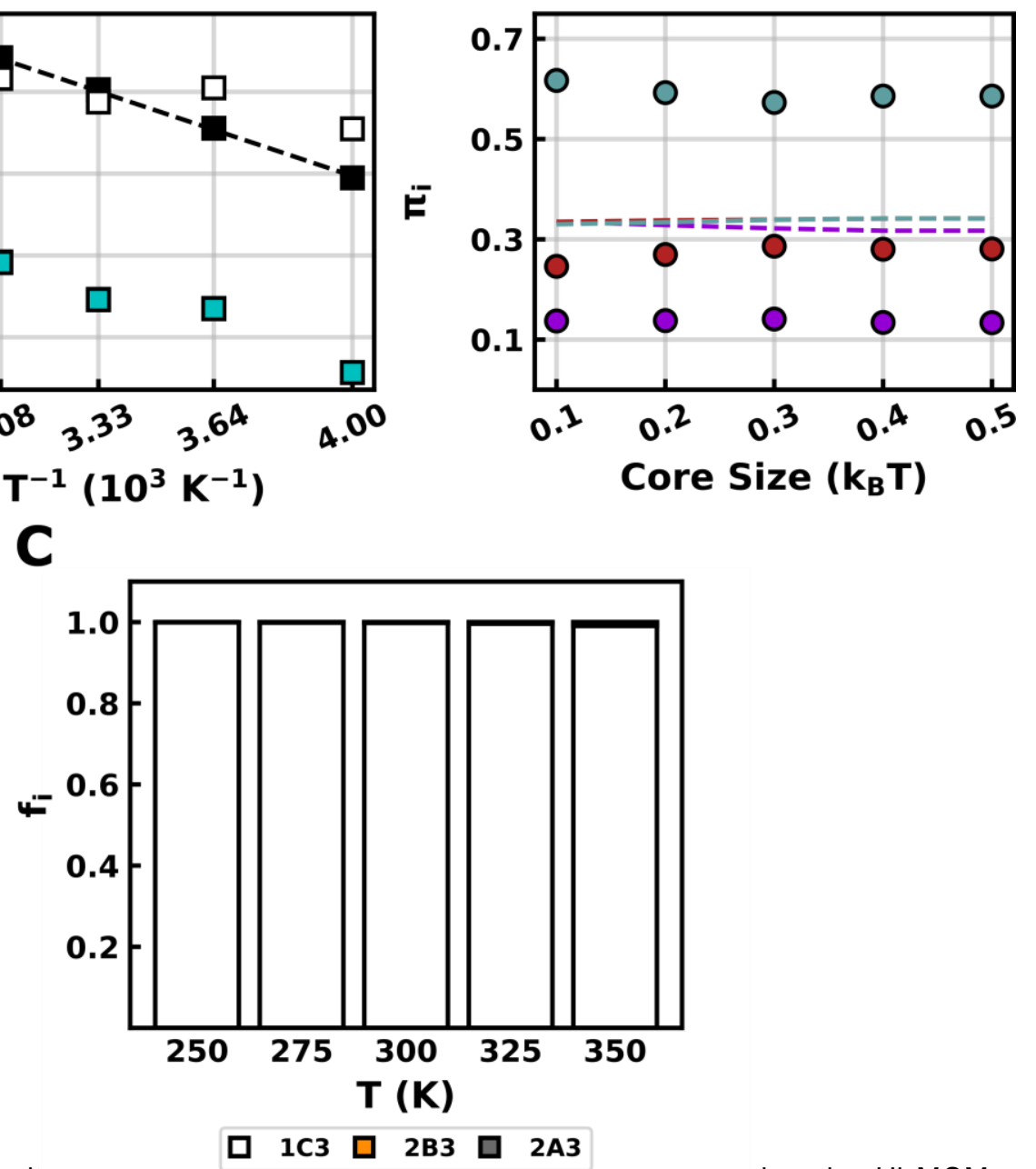

Figure S5. Hi-MSM result

(blue), the Hi-MSM with only path 1C3 (cyan), and the single pathway (1C3 only) results (white) to the unbiased simulations (black). The blue and cyan traces overlap. The results shown are for the Hi-MSM constructed with a core size of $0.3 k_{\mathrm{B}} T$. (B) The probability of occupying states 1-3 at equilibrium calculated with the Hi-MSM (circles) compared to the simulation results (dashed lines added to guide the eye). The color scheme for states 1-3 matches those in Figure 3B. Results are shown for the model constructed at $300 \mathrm{~K}$. (C) Fraction of the flux from $\Lambda_{1} \rightarrow \Lambda_{2}$ from the Hi-MSM for individual pathways. The results are displayed for the models constructed at $0.3 k_{\mathrm{B}} T$. Pathway labels correspond to the Markov and transition state labels shown in Figure S2. The error bars, which are too small to be visible, represent the $95 \%$ confidence intervals obtained from bootstrapping the underlying rate matrices. 


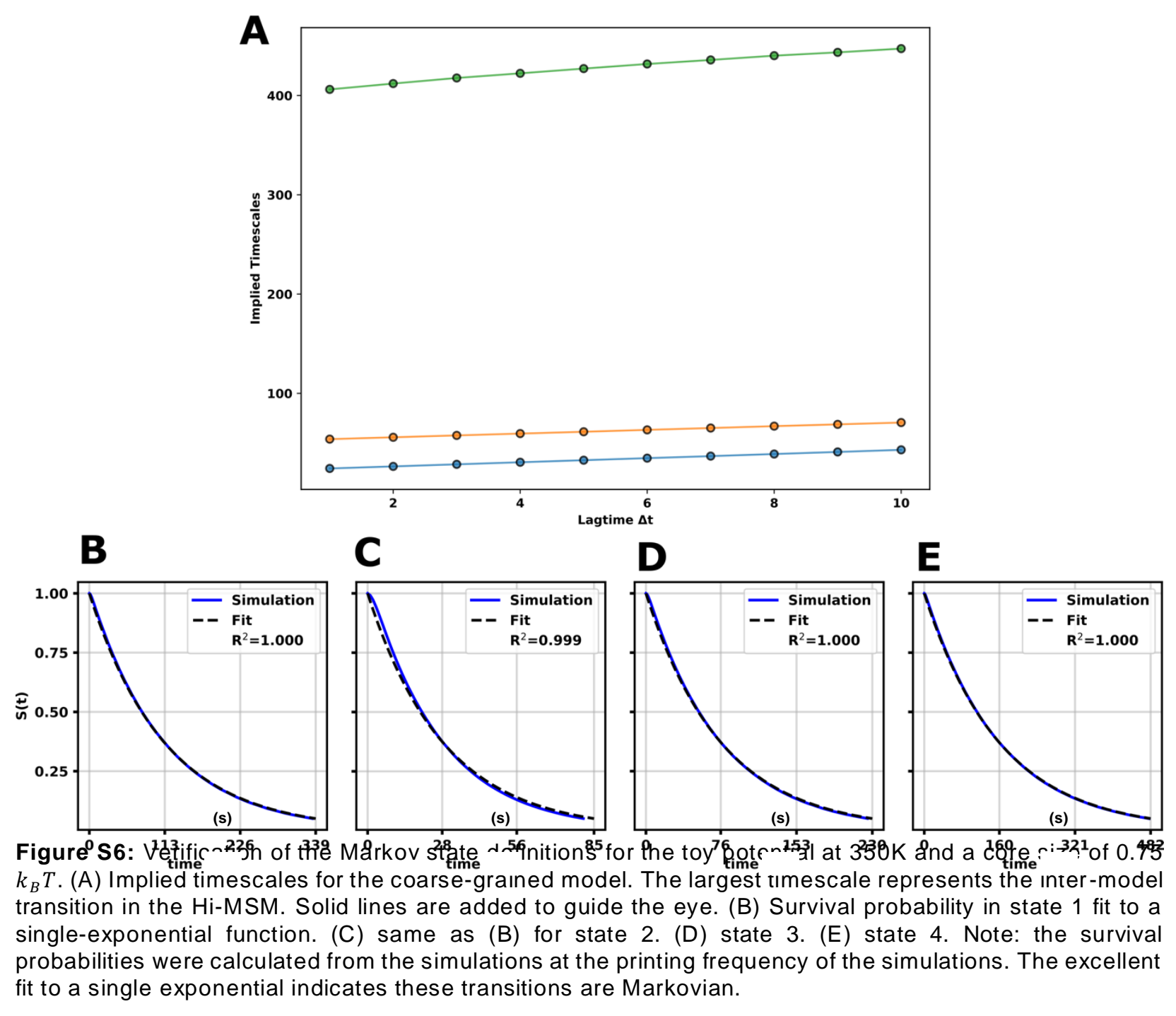



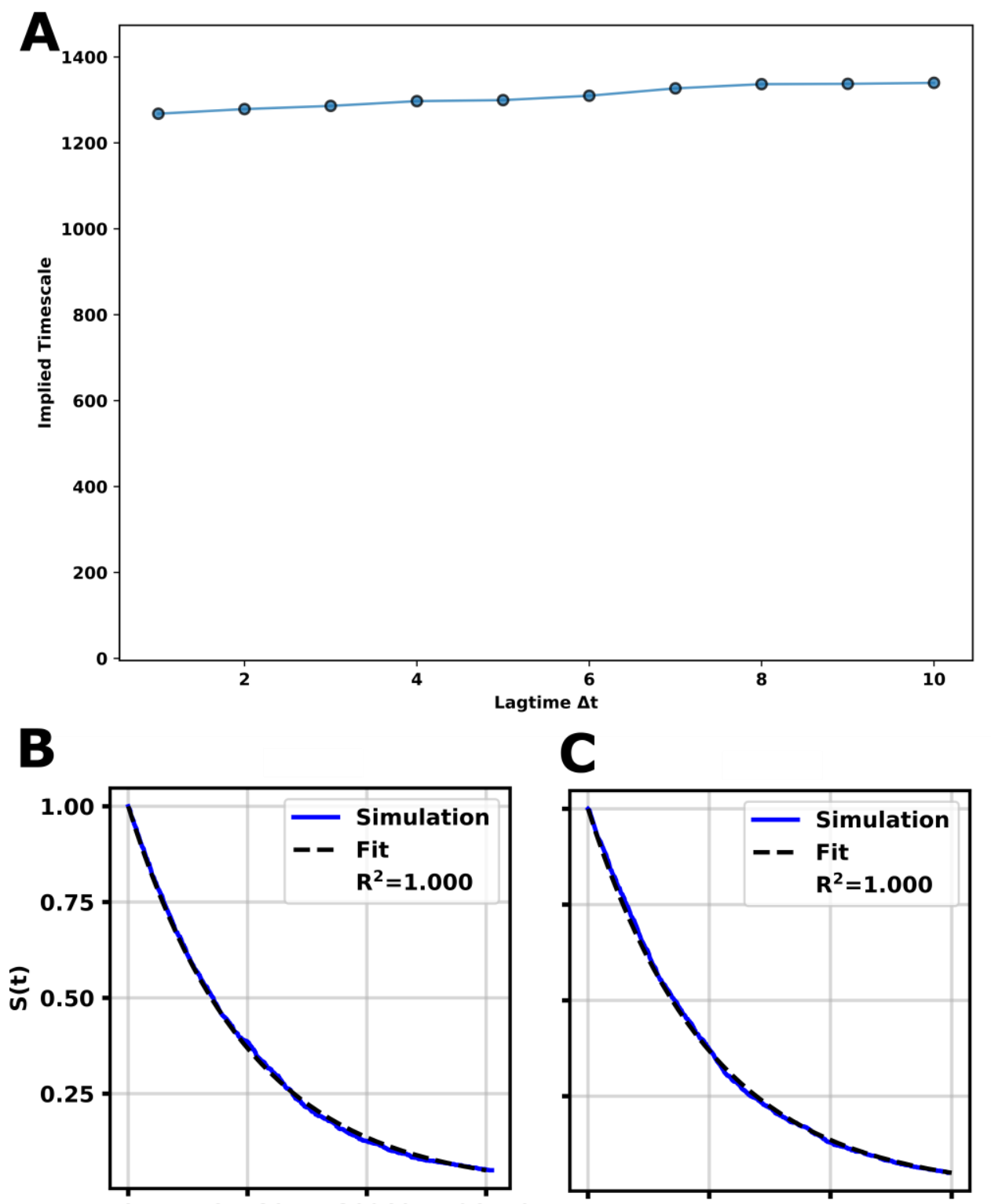

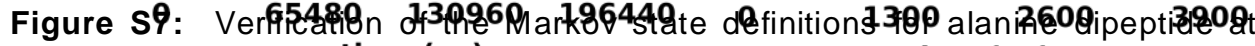

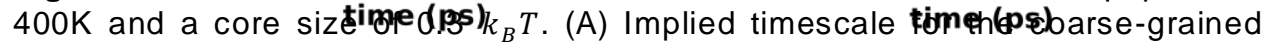
model. This timescale represents the transition between local MSMs. (B) Survival probability in state 1 fit to a single-exponential function. (C) same as (B) for state 4. Note: the survival probability was calculated using the printing frequency of the simulations. The excellent fit to a single exponential indicates these transitions are Markovian. 

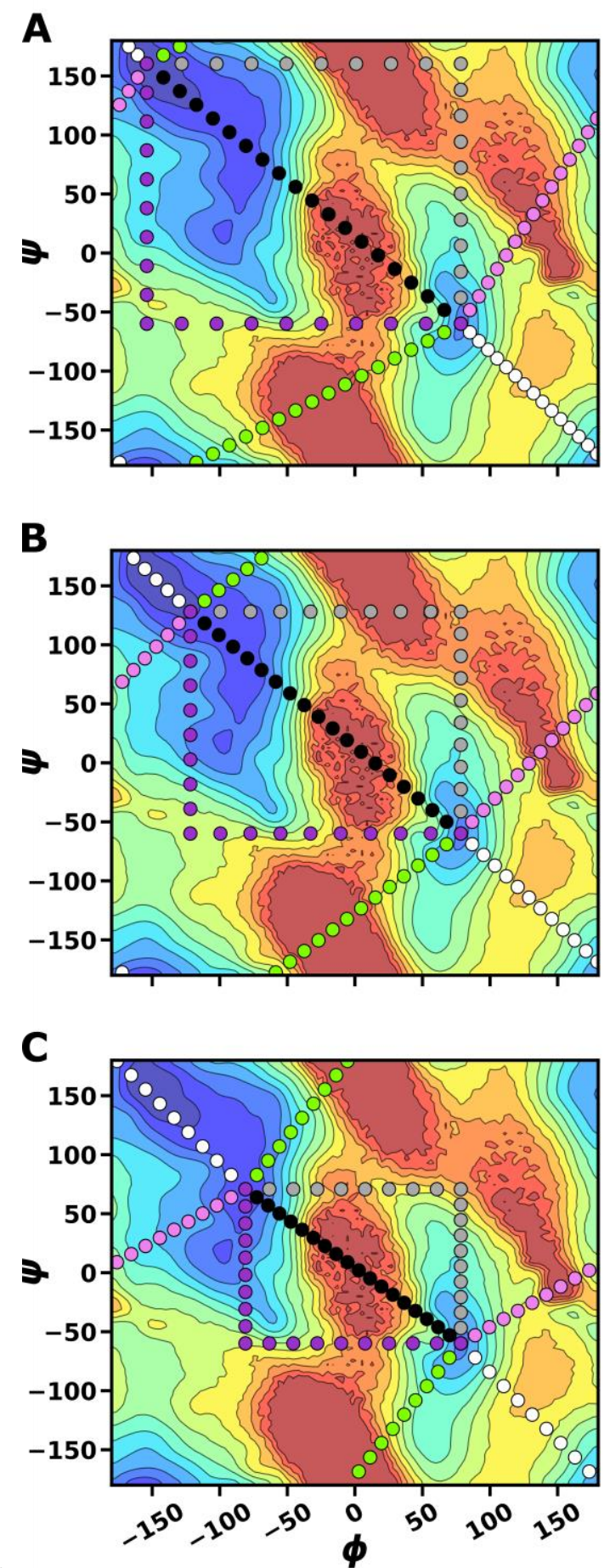

Figure S8. Initial guesses for . . .... . . n....... $2 \rightarrow 4$, C) $3 \rightarrow 4$. 

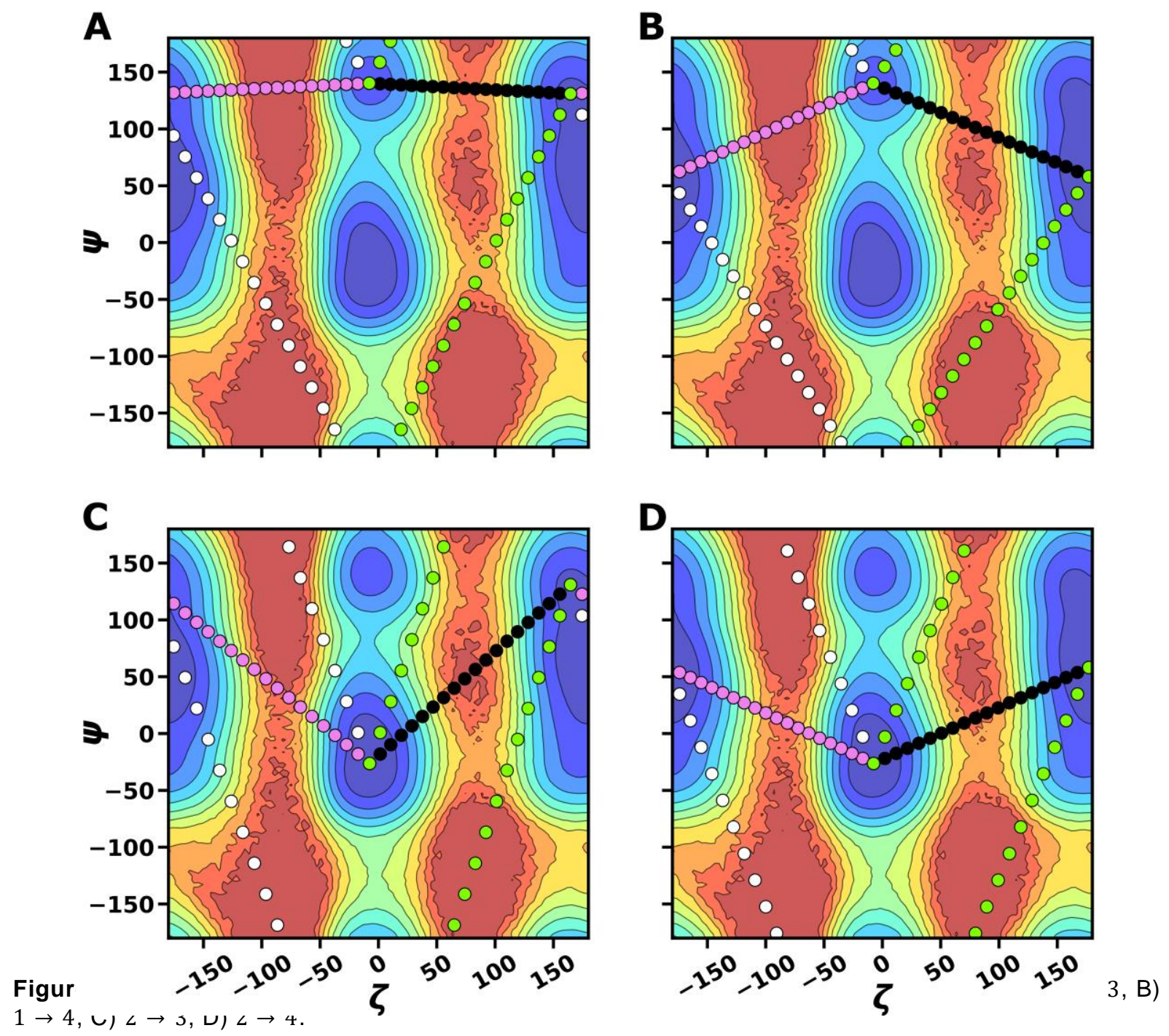

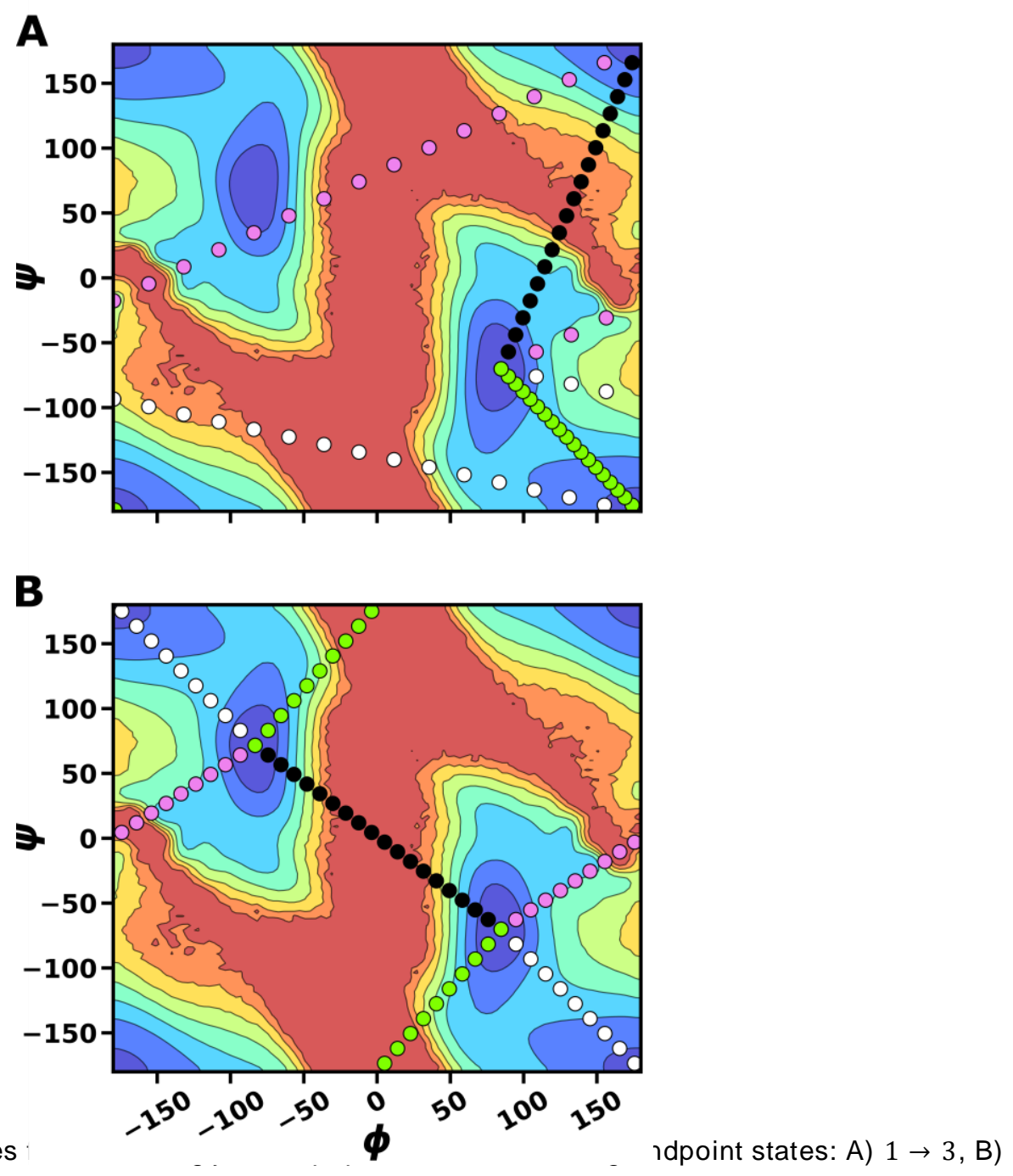

$2 \rightarrow 3$ 
Table S1. Comparison of the Average Percent Errors in Predicted Quantities using the Hi-MSM and Single-Path Methods for Proline Dipeptide ${ }^{d}$

$\begin{array}{lcccc} & <\text { Error } \boldsymbol{k}_{\boldsymbol{o b s}}> & <\text { Error } \boldsymbol{\pi}_{\boldsymbol{i}}>^{a} & <\text { Error } \boldsymbol{f}_{\boldsymbol{o b s}}>^{\boldsymbol{b}} & \text { Error } \boldsymbol{m}^{\boldsymbol{c}} \\ \text { Hi-MSM } & 9.0 \pm 0.5 & 11.6 \pm 0.8 & 16.5 \pm 0.4 & 2.60 \\ \text { Hi-MSM 1 path } & 64.8 \pm 0.5 & 60.5 \pm 0.8 & 89.9 \pm 0.6 & 14.8 \\ \text { Single Path } & 58.7 \pm 0.5 & - & 86.4 \pm 0.4 & 12.1 \\ { }^{a} \pi_{i} \text { is the probability of being in state } \mathrm{i} & \\ { }^{b} f_{\text {obs }} \text { is the total observed flux from } \Lambda_{1} \rightarrow \Lambda_{2} \\ { }^{c} m \text { is the slope of the Arrhenius plot } \\ { }^{d} \text { Reported uncertainties represent the 95\% confidence interval. }\end{array}$

Table S2. Comparison of the Average Percent Errors in Predicted Quantities using the Hi-MSM and Single Path Methods for Glycine Dipeptide ${ }^{d}$

\begin{tabular}{|c|c|c|c|c|}
\hline & $<$ Error $\boldsymbol{k}_{\text {obs }}>$ & $<$ Error $\pi_{i}>^{a}$ & $<$ Error $f_{o b s}>^{b}$ & Error $\boldsymbol{m}^{c}$ \\
\hline Hi-MSM & $71.03 \pm 0.05$ & $49.25 \pm 0.10$ & $81.94 \pm 0.00$ & 24.3 \\
\hline Hi-MSM 1 path & $71.14 \pm 0.04$ & $49.48 \pm 0.10$ & $82.06 \pm 0.00$ & 25.3 \\
\hline Single Path & $22.73 \pm 0.06$ & - & $72.30 \pm 0.00$ & 77.0 \\
\hline
\end{tabular}

Table S3. Percent Errors in Predicted Rates versus Core Size for Proline Dipeptide at 700K. Reported Uncertainties Represent the 95\% Confidence Interval.

\begin{tabular}{cccc} 
& \multicolumn{3}{c}{ model } \\
\cline { 2 - 4 } core size $\left(\boldsymbol{k}_{\mathrm{B}} \boldsymbol{T}\right)$ & Hi-MSM & Hi-MSM 1 path & single path \\
0.1 & $13.21 \pm 0.49$ & $67.11 \pm 0.47$ & $61.44 \pm 0.48$ \\
0.2 & $18.92 \pm 0.51$ & $73.85 \pm 0.48$ & $69.35 \pm 0.49$ \\
0.3 & $9.00 \pm 0.50$ & $64.78 \pm 0.48$ & $58.73 \pm 0.49$ \\
0.4 & $21.03 \pm 0.51$ & $67.00 \pm 0.47$ & $61.29 \pm 0.48$ \\
0.5 & $20.99 \pm 0.51$ & $67.68 \pm 0.48$ & $62.07 \pm 0.48$
\end{tabular}


Table S4. Percent Errors in Predicted Rates versus Core Size for Glycine Dipeptide at 300K. Reported Uncertainties Represent the 95\% Confidence Interval.

\begin{tabular}{cccc} 
& \multicolumn{3}{c}{ model } \\
\cline { 2 - 4 } core size $\left(\boldsymbol{k}_{\mathrm{B}} \boldsymbol{T}\right)$ & Hi-MSM & Hi-MSM 1 path & single path \\
0.1 & $61.53 \pm 0.05$ & $61.68 \pm 0.04$ & $21.49 \pm 0.06$ \\
0.2 & $68.67 \pm 0.05$ & $68.78 \pm 0.04$ & $20.43 \pm 0.06$ \\
0.3 & $71.03 \pm 0.05$ & $71.14 \pm 0.04$ & $22.73 \pm 0.06$ \\
0.4 & $71.48 \pm 0.05$ & $71.58 \pm 0.04$ & $22.87 \pm 0.06$ \\
0.5 & $73.01 \pm 0.05$ & $73.12 \pm 0.04$ & $15.14 \pm 0.05$
\end{tabular}

Table S5. Alanine Dipeptide FTSM Parameters at 400K

\begin{tabular}{|c|c|c|c|c|c|}
\hline Temperature (K) & Cutoff $\left(k_{B} T\right)$ & Path Label & $\operatorname{kpar}[\AA ̊]$ & $\operatorname{kprp}[\AA]]$ & dprp $[\AA]]$ \\
\hline 400 & 0.1 & $1 C 4$ & 7979 & 6839 & 0.1 \\
\hline 400 & 0.1 & $1 \mathrm{~A} 4$ & 9867 & 3524 & 0.12 \\
\hline 400 & 0.1 & 1D4 & 7979 & 6839 & 0.1 \\
\hline 400 & 0.1 & $1 \mathrm{E} 4$ & 9867 & 3524 & 0.12 \\
\hline 400 & 0.2 & $1 \mathrm{~A} 4$ & 9867 & 3524 & 0.12 \\
\hline 400 & 0.2 & 1FE4 & 12500 & 5000 & 0.1 \\
\hline 400 & 0.2 & $1 C 4$ & 7979 & 6839 & 0.1 \\
\hline 400 & 0.2 & 1E4 & 7979 & 6839 & 0.1 \\
\hline 400 & 0.2 & 1D4 & 7979 & 6839 & 0.1 \\
\hline 400 & 0.3 & $1 \mathrm{E} 4$ & 7979 & 6839 & 0.1 \\
\hline 400 & 0.3 & $1 C 4$ & 7979 & 6839 & 0.1 \\
\hline 400 & 0.3 & 1FE4 & 12500 & 5000 & 0.1 \\
\hline 400 & 0.3 & 1D4 & 7979 & 6839 & 0.1 \\
\hline 400 & 0.3 & $1 \mathrm{~A} 4$ & 9867 & 3524 & 0.12 \\
\hline 400 & 0.4 & 1D4 & 7979 & 6839 & 0.1 \\
\hline 400 & 0.4 & $1 C 4$ & 7979 & 6839 & 0.1 \\
\hline 400 & 0.4 & $1 \mathrm{E} 4$ & 7979 & 6839 & 0.1 \\
\hline 400 & 0.4 & 1FE4 & 12500 & 5000 & 0.1 \\
\hline 400 & 0.4 & $1 \mathrm{~A} 4$ & 9867 & 3524 & 0.12 \\
\hline
\end{tabular}


Table S6. Alanine Dipeptide FTSM Parameters at 450K

\begin{tabular}{|c|c|c|c|c|c|}
\hline Temperature (K) & Cutoff $\left(k_{B} T\right)$ & Path Label & $\operatorname{kpar}[\AA ̊]$ & $\operatorname{kprp}[\AA]$ & dprp [Å] \\
\hline 450 & 0.1 & 1FE4 & 12500 & 5000 & 0.1 \\
\hline 450 & 0.1 & $1 \mathrm{~A} 4$ & 9867 & 5000 & 0.12 \\
\hline 450 & 0.1 & $3 C 4$ & 4252 & 3307 & 0.18 \\
\hline 450 & 0.1 & $3 \mathrm{~A} 4$ & 8128 & 4000 & 0.1 \\
\hline 450 & 0.1 & 1D4 & 10000 & 6839 & 0.1 \\
\hline 450 & 0.1 & 3AB4 & 15000 & 10000 & 0.1 \\
\hline 450 & 0.2 & $3 C 4$ & 4252 & 3307 & 0.18 \\
\hline 450 & 0.2 & 3AB4 & 15000 & 10000 & 0.1 \\
\hline 450 & 0.2 & 1FE4 & 12500 & 5000 & 0.1 \\
\hline 450 & 0.2 & $3 \mathrm{E} 4$ & 9867 & 5000 & 0.12 \\
\hline 450 & 0.2 & 1D4 & 10000 & 6839 & 0.1 \\
\hline 450 & 0.2 & $3 A 4$ & 8128 & 4000 & 0.1 \\
\hline 450 & 0.2 & 1A4 & 9867 & 5000 & 0.12 \\
\hline 450 & 0.3 & 1A4 & 9867 & 5000 & 0.12 \\
\hline 450 & 0.3 & $3 \mathrm{E} 4$ & 8128 & 4000 & 0.1 \\
\hline 450 & 0.3 & 3AB4 & 15000 & 10000 & 0.1 \\
\hline 450 & 0.3 & 1FE4 & 12500 & 5000 & 0.1 \\
\hline 450 & 0.3 & $3 C 4$ & 4252 & 3307 & 0.18 \\
\hline 450 & 0.3 & $3 A 4$ & 8128 & 4000 & 0.1 \\
\hline 450 & 0.3 & 1D4 & 10000 & 6839 & 0.1 \\
\hline 450 & 0.4 & 1FE4 & 12500 & 5000 & 0.1 \\
\hline 450 & 0.4 & $3 C 4$ & 4252 & 3307 & 0.18 \\
\hline 450 & 0.4 & 3AB4 & 15000 & 10000 & 0.1 \\
\hline 450 & 0.4 & 1D4 & 10000 & 6839 & 0.1 \\
\hline 450 & 0.4 & $3 \mathrm{E} 4$ & 9867 & 5000 & 0.12 \\
\hline 450 & 0.4 & 1A4 & 9867 & 5000 & 0.12 \\
\hline 450 & 0.4 & $3 \mathrm{~A} 4$ & 8128 & 4000 & 0.1 \\
\hline
\end{tabular}

Table S7. Alanine Dipeptide FTSM Parameters at 500K

\begin{tabular}{|c|c|c|c|c|c|}
\hline Temperature (K) & Cutoff $\left(k_{B} T\right)$ & Path Label & $\operatorname{kpar}[\AA]$ & $\operatorname{kprp}[\AA ̊]$ & $\operatorname{dprp}[\AA]$ \\
\hline 500 & 0.1 & $3 C 4$ & 4252 & 3307 & 0.18 \\
\hline 500 & 0.1 & 1D4 & 10000 & 6839 & 0.1 \\
\hline 500 & 0.1 & $3 \mathrm{AB} 4$ & 12000 & 5000 & 0.12 \\
\hline 500 & 0.1 & $3 \mathrm{E} 4$ & 8128 & 4000 & 0.12 \\
\hline 500 & 0.1 & $2 \mathrm{~A} 4$ & 8128 & 4000 & 0.12 \\
\hline 500 & 0.1 & 1FE4 & 18000 & 5000 & 0.13 \\
\hline 500 & 0.2 & 1FE4 & 18000 & 5000 & 0.13 \\
\hline 500 & 0.2 & 1D4 & 10000 & 6839 & 0.1 \\
\hline 500 & 0.2 & $3 \mathrm{E} 4$ & 8128 & 4000 & 0.12 \\
\hline 500 & 0.2 & $3 C 4$ & 4252 & 3307 & 0.18 \\
\hline 500 & 0.2 & $3 \mathrm{~A} 4$ & 8128 & 4000 & 0.12 \\
\hline 500 & 0.2 & $2 \mathrm{~A} 4$ & 8128 & 4000 & 0.12 \\
\hline 500 & 0.2 & $3 \mathrm{AB} 4$ & 12000 & 5000 & 0.12 \\
\hline 500 & 0.3 & 1FE4 & 18000 & 5000 & 0.13 \\
\hline 500 & 0.3 & $3 \mathrm{AB} 4$ & 12000 & 5000 & 0.12 \\
\hline 500 & 0.3 & 1D4 & 10000 & 6839 & 0.1 \\
\hline 500 & 0.3 & $3 C 4$ & 4252 & 3307 & 0.18 \\
\hline 500 & 0.3 & $2 \mathrm{~A} 4$ & 8128 & 4000 & 0.12 \\
\hline 500 & 0.3 & $3 \mathrm{E} 4$ & 8128 & 4000 & 0.12 \\
\hline 500 & 0.3 & $3 \mathrm{~A} 4$ & 8128 & 4000 & 0.12 \\
\hline 500 & 0.4 & 3AB4 & 12000 & 5000 & 0.12 \\
\hline 500 & 0.4 & $3 \mathrm{E} 4$ & 8128 & 4000 & 0.12 \\
\hline 500 & 0.4 & 1FE4 & 18000 & 5000 & 0.13 \\
\hline 500 & 0.4 & $3 \mathrm{~A} 4$ & 8128 & 4000 & 0.12 \\
\hline 500 & 0.4 & $3 C 4$ & 4252 & 3307 & 0.18 \\
\hline 500 & 0.4 & $2 \mathrm{~A} 4$ & 8128 & 4000 & 0.12 \\
\hline 500 & 0.4 & 1D4 & 10000 & 6839 & 0.1 \\
\hline
\end{tabular}


Table S8. Alanine Dipeptide FTSM Parameters at 550K

\begin{tabular}{|c|c|c|c|c|c|}
\hline Temperature (K) & Cutoff $\left(k_{B} T\right)$ & Path Label & $\operatorname{kpar}[\AA]$ & kprp [Å] & dprp [Å] \\
\hline 550 & 0.1 & 3E4 & 8128 & 4000 & 0.12 \\
\hline 550 & 0.1 & 3AB4 & 30000 & 10000 & 0.1 \\
\hline 550 & 0.1 & $3 C 4$ & 8000 & 4000 & 0.12 \\
\hline 550 & 0.1 & $3 \mathrm{~A} 4$ & 8128 & 4000 & 0.1 \\
\hline 550 & 0.1 & 1D4 & 10000 & 6839 & 0.1 \\
\hline 550 & 0.1 & 1FE4 & 10000 & 6839 & 0.1 \\
\hline 550 & 0.2 & $3 C 4$ & 8000 & 4000 & 0.12 \\
\hline 550 & 0.2 & 3AB4 & 30000 & 10000 & 0.1 \\
\hline 550 & 0.2 & $3 \mathrm{~A} 4$ & 8128 & 4000 & 0.1 \\
\hline 550 & 0.2 & 1D4 & 10000 & 6839 & 0.1 \\
\hline 550 & 0.2 & $3 \mathrm{E} 4$ & 8000 & 4000 & 0.12 \\
\hline 550 & 0.2 & $2 \mathrm{~A} 4$ & 8000 & 5000 & 0.12 \\
\hline 550 & 0.2 & 1FE4 & 10000 & 6839 & 0.1 \\
\hline 550 & 0.3 & $3 E 4$ & 8128 & 4000 & 0.12 \\
\hline 550 & 0.3 & 1D4 & 10000 & 6839 & 0.1 \\
\hline 550 & 0.3 & $3 \mathrm{~A} 4$ & 8128 & 4000 & 0.1 \\
\hline 550 & 0.3 & $2 \mathrm{~A} 4$ & 8000 & 5000 & 0.12 \\
\hline 550 & 0.3 & 3AB4 & 30000 & 10000 & 0.1 \\
\hline 550 & 0.3 & $3 C 4$ & 8000 & 4000 & 0.12 \\
\hline 550 & 0.4 & $3 C 4$ & 8000 & 4000 & 0.12 \\
\hline 550 & 0.4 & $2 \mathrm{~A} 4$ & 8000 & 5000 & 0.12 \\
\hline 550 & 0.4 & 1D4 & 10000 & 6839 & 0.1 \\
\hline 550 & 0.4 & $3 \mathrm{E} 4$ & 8128 & 4000 & 0.12 \\
\hline
\end{tabular}

Table S9. Alanine Dipeptide FTSM Parameters at $600 \mathrm{~K}$

\begin{tabular}{|c|c|c|c|c|c|}
\hline Temperature (K) & Cutoff $\left(k_{B} T\right)$ & Path Label & $\operatorname{kpar}[\AA]]$ & $\operatorname{kprp}[\AA]]$ & dprp $[\AA]]$ \\
\hline 600 & 0.1 & $3 \mathrm{E} 4$ & 7935 & 4534 & 0.1 \\
\hline 600 & 0.1 & $3 C 4$ & 10000 & 5000 & 0.1 \\
\hline 600 & 0.1 & $2 \mathrm{~A} 4$ & 8000 & 4000 & 0.1 \\
\hline 600 & 0.1 & 1D4 & 7979 & 6839 & 0.1 \\
\hline 600 & 0.1 & $3 \mathrm{~A} 4$ & 7935 & 4534 & 0.1 \\
\hline 600 & 0.2 & $3 C 4$ & 10000 & 5000 & 0.1 \\
\hline 600 & 0.2 & 1D4 & 7979 & 6839 & 0.1 \\
\hline 600 & 0.2 & $3 \mathrm{E} 4$ & 7935 & 4534 & 0.1 \\
\hline 600 & 0.2 & $2 \mathrm{~A} 4$ & 8000 & 4000 & 0.1 \\
\hline 600 & 0.3 & 1D4 & 7979 & 6839 & 0.1 \\
\hline 600 & 0.3 & $3 C 4$ & 10000 & 5000 & 0.1 \\
\hline 600 & 0.3 & $2 \mathrm{~A} 4$ & 8000 & 4000 & 0.1 \\
\hline 600 & 0.3 & $3 \mathrm{E} 4$ & 7935 & 4534 & 0.1 \\
\hline 600 & 0.4 & $2 \mathrm{~A} 4$ & 8000 & 4000 & 0.1 \\
\hline 600 & 0.4 & $3 C 4$ & 10000 & 5000 & 0.1 \\
\hline 600 & 0.4 & $3 E 4$ & 7935 & 4534 & 0.1 \\
\hline 600 & 0.4 & 1D4 & 7979 & 6839 & 0.1 \\
\hline
\end{tabular}

